\title{
In Situ Inspection for Generator Retaining Rings of Geothermal Power Plant $60 \mathrm{MW}$
}

\author{
Imam Djunaedi and Hilman Syaeful Alam
}

\begin{abstract}
The structural integrity of generator retaining ring at Dieng geothermal power plant $60 \mathrm{MW}$ has been investigated using NDT method, i.e. visual testing, penetrant testing, ultrasonic testing and crack depth testing. The purpose of this study is to evaluate the integrity of retaining ring without removing the retaining ring from the rotor, because releasing the retaining ring from the generator rotor will eliminate residual stresses therefore the defects are not detected due to residual stress. In addition, this operation has major implications of damage risk, cost, safety and time. The results of the inspection of the above method was not detected the presence of defects on both sides of the retaining ring generator (turbine and exciter side). Therefore, it can be concluded that the retaining ring is not degraded and the service life of components is still appropriate with the specifications of the manufacturer.
\end{abstract}

Index Terms-Generator retaining ring, structural integrity, NDT inspection, defect.

\section{INTRODUCTION}

Retaining ring is one of the generator components that used to retain the end winding coil assembly, insulation material and packing blocks against centrifugal forces developed when the rotor is rotating that are shown in Fig. 1. Therefore it is the most highly stressed component of the generator and possibly the highest stressed component of the entire turbine generator set, including both steam turbines and combustion turbines [1]. The shrink fit method by thermal expansion and contraction of metal is used to mount the retaining ring on the generator rotor. According to interference of shrink fit, contact pressure is changed and residual stresses appear to fit the components. Excessive residual stress causes a defect in products and stress corrosion cracking, SCC [2]. The contact separation and failure occurrences for those common systems have been more rarely addressed in the literature [3]-[12]. Under cyclic bending or torsion loads, fretting could occur at the edge of the shaft-hub fitting surface. Fretting is a wear/corrosion mechanism resulting from the slight relative movement of contacting surfaces [9].

Manuscript received May 10, 2014; revised August 23, 2014. This work was supported in part by the Indonesian Institute of Sciences and PT. Geo Dipa Energy (Dieng Geothermal Power Plant).

Imam Djunaedi is with Research Center for Physics, Indonesian Institute of Sciences, Jl. Sangkuriang Komp. LIPI Gd. 80, Bandung, 40135, Indonesia (e-mail: imam_djunaedi@yahoo.com).

Hilman Syaeful Alam is with the Technical Implementation Unit for Instrumentation Development, Indonesian Institute of Sciences, Jl. Sangkuriang Komp. LIPI Gd. 30, Bandung, 40135, Indonesia (e-mail: hilm003@lipi.go.id).

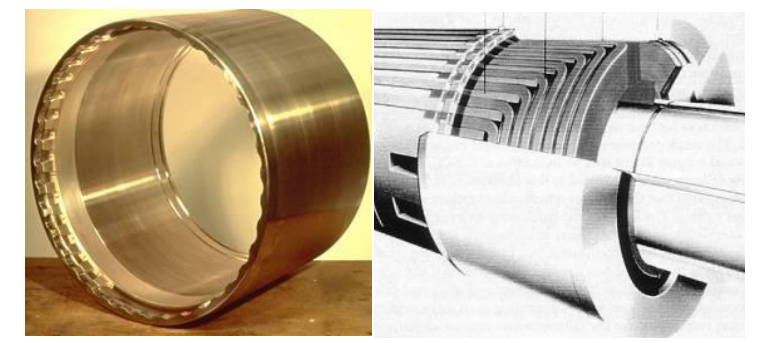

Fig. 1. Typical turbine generator end-winding retaining ring [1], [11].

In the case of generator retaining rings, fretting can develop at shrink fit and mating surfaces and at winding support positions. It can also suffer damage caused by torsional vibration. Fretting fatigue initiated crack that propagated during torsional interaction of the shaft [10]. The high levels of residual stress from the cold working process and further loads which are imposed by shrink fitting, self-weight and copper support loads during operation can lead to service failure. The service failures on the retaining ring are known to result in catastrophic failure of the generator and part of its associated turbine or prime mover. It is possible for these rings to rupture or "explode" damaging the stator end winding, rotor winding and end cover that is shown in Fig. 2 [1].

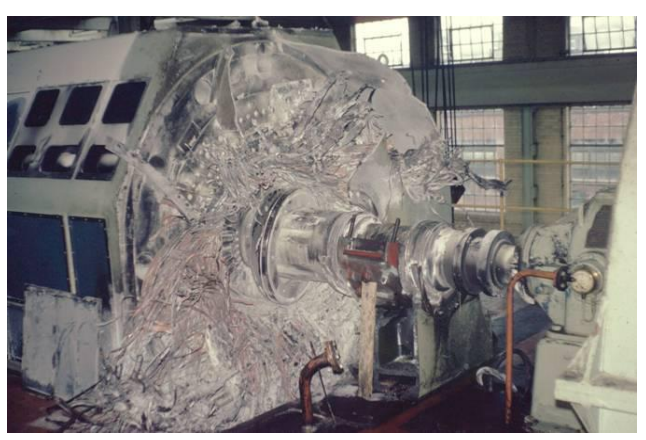

Fig. 2. Generator retaining ring failure [1].

\section{MATERIALS AND METHODS}

Dieng is one of the geothermal power plants in Indonesia that is located in Dieng Plateau, under the administrative district of Wonosobo, Central Java, Indonesia. The plant was installed in 1995 with rating generator $75000 \mathrm{KVA}$, Voltage 15000 Volts, Current 2887 Amp, and Rpm 3000. The generator rotor has two poles of winding, both leads position on the exciter side disconnected to the diodes and exciter armature. In 2013, the plant was reportedly suffered damage due to malfunction of the flow control system causing the over flow of working fluid. There are a damage to some components, i.e. the turbine and generator rotor. In the generator rotor, damage occurs on the bearing and labyrinth seal, which is thought to be caused by high vibration due to 
over speed. In addition these components damages, it is feared the damage also occurred in the generator retaining ring.

Traditionally, the inspections of retaining ring flaws at internal ring bore locations which cause the failures require the removal of the retaining ring from the rotor. This operation has major implications of damage risk, cost, safety and timing to an outage program. To overcome this problem and achieve significant savings, the integrity of retaining ring is evaluated using powerful inspection technique based on Non Destructive (NDT) inspection. This technique is enable fast and accurate inspection results, with the retaining ring remains patches to its rotor. The advantages of this method compared to removal the ring from the rotor are the stresses from the shrink fit helps to keep any more cracks open and easier to detect. It was reported a case where cracks were detected while the ring was installed but could not be detected once removed [1]. In this study, we use several NDT methods, i.e. Visual Testing, Penetrant Testing with Crack Depth Meter, and Ultrasonic Testing.

\section{A. Visual Testing}

The ring is inspected visually for any signs of mechanical damage, corrosion, pitting, heat damage, electrical arcing marks, fretting, rusting staining or cracking. If any of the above defects is observed it has to be evaluated to determine its severity, consequences and root cause. Rusting, pitting or staining is an indication that the rings have been exposed to a corrosive environment and careful attention needs to be paid to the possibility of stress corrosion cracking being present. Mechanical damage is normally groundout in order to expose any defects. A visual inspection on its own is not sufficient and hence a more detailed inspection below is required.

\section{B. Dye Penetrant Testing (PT)}

For the outer surface of the retaining ring, pitting and cracking would be detected by using a liquid dye penetrant. Testing is done by applying dye penetrant on the surface of the samples analyzed in a given time interval (dwell time). During that interval, the liquid penetrant defect entered on the surface, the penetrant which is not entered is cleaned. Further, sprays or powders contrast color developer is used to describe the profile of defects on the material. The results of these operations are then evaluated against acceptance criteria: if the criteria are not transgressed the ring is deemed acceptable and no further action is taken.

\section{Crack Depth Testing}

Inspection using the PT cannot exactly figure out the depth of a flaw or crack, so the crack depth gauge is used to determine the depth of defect, as shown in Fig. 3. Crack depth gauge is used to measure crack depth by applying principle of acoustic diffraction. It also can be used to measure propagation velocity of ultrasonic wave in retaining ring. This instrument is a kind of intelligent nondestructive test equipment possessing the functions of automatic detection, data memory and output.

\section{Ultrasonic Testing (UT)}

Ultrasonic Testing would be used for detecting the flaws at the inner surface of the retaining ring. UT can be scanning for both axial and circumferential flaws, specific geometry (bayonet area, et cetera), determining wall thickness, confirming flaw indications, mapping of small pitting resulting from corrosion or electrical discharges, and mapping of ring geometry / teeth in shrink fit connection. There are several advantages to using the UT method, i.e. reliable and accurate defect height sizing, detects all cracks including the ones that are out of the perpendicular plane, used for confirmation of surface breaking indications on the inner surface, and used for "difficult" areas: at (undulated) bayonet structure; at grooved areas at a ring's end.

The working principle of ultrasonic testing is the use of ultrasonic waves, see Fig. 4. Ultrasonic wavelengths are on the same order of magnitude as visible light, giving them many of the same properties of light. For example, ultrasonic wavelengths can be focused, reflected, and refracted. Ultrasonic waves are transmitted through air, water, and solids such as steel by high-frequency particle vibrations. These waves are transmitted in homogenous solid objects much like pointing a flashlight around a room with various objects that reflect light. The directed energy in an ultrasonic wave is reflected by boundaries between materials regardless of whether the material is gas, liquid, or solid. Ultrasonic waves are also reflected by any cracks or voids in solid materials. These reflected waves, which are caused by internal defects, can be compared to the reflected waves from the external surfaces, enabling the size and severity of internal defects to be identified.

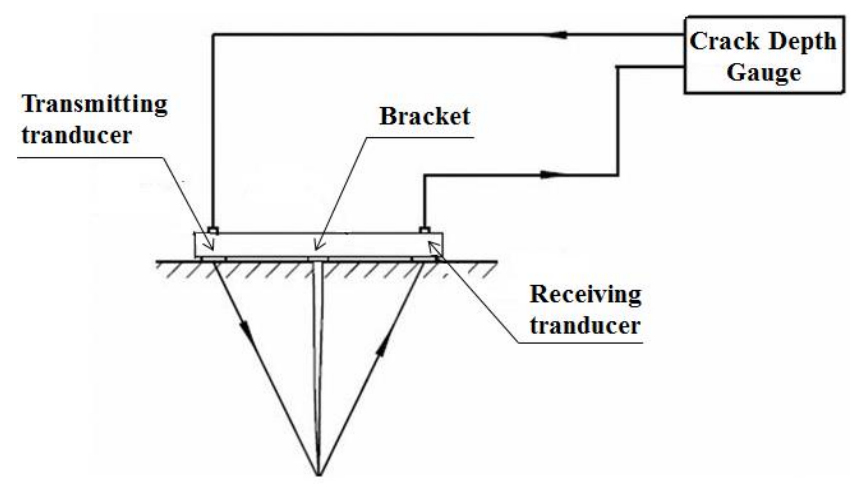

Fig. 3. Crack depth gauge [13].

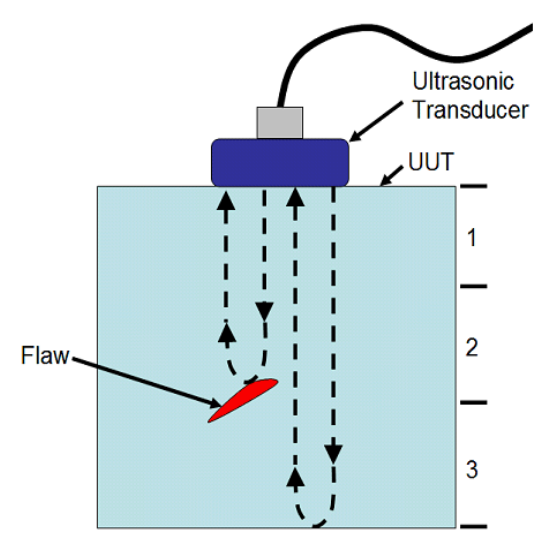

Fig. 4. The working principle of ultrasonic testing [14]

The ultrasonic testing is range from $400 \mathrm{kHz}$ to $25 \mathrm{MHz}$. The frequency of the ultrasonic sensor is chosen based on several factors including detectable flaw size, depth of penetration, and grain size of the material. Materials made of fine-grained material, such as metals, permit deep penetration by ultrasonic waves of all frequencies. The higher the frequency, the smaller the flaws the system detects, but the 
depth of penetration decreases. Fig. 5 displays of ultrasonic testing to detect the presence of a crack in a component.

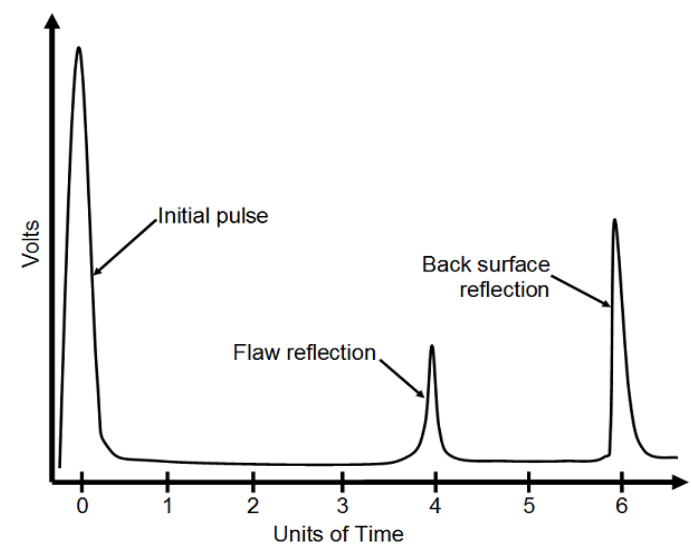

Fig. 5. The display of ultrasonic testing to detect the presence of a crack in a component [14].

\section{RESULTS AND DISCUSSIONS}

Based on the visual inspection, it didn't find any damage on the surface of the retaining ring at the both side i.e. exciter side and turbine side. Fig. 6 shows the surface condition of the retaining ring which is visually no visible signs of a flaw or defect. To further validate the structural integrity of the retaining ring, the inspection is done using advanced NDT methods.

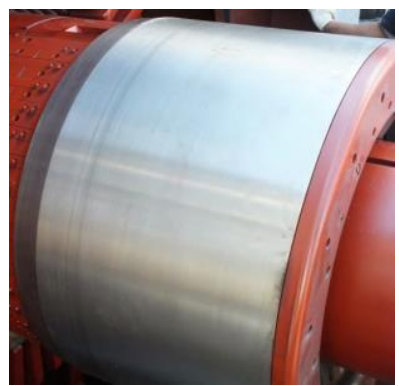

Fig. 6. No marks on retaining ring surface

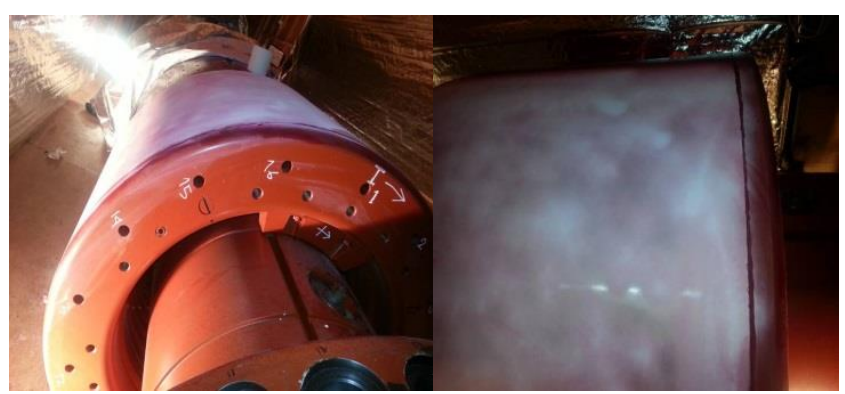

(a)

(b)

Fig. 7. PT inspection results for (a) exciter side and (b) generator side.

The penetrant testing (PT) refers to non flourescent or solvent removable method. It was done by applying a dye penetrant on the surface of retaining ring at 5 minutes of dwell time. The developer is used to describe the profile of defects on the material. In this study, it is used the SKL-SP type of penetrant from Magnaflux with SKD-2 developer type and SKC-S remover type. Based on this inspection, it was not detected the presence of defects on both sides of the retaining ring generator. Fig. 7 shows the PT inspection result of the retaining ring for turbine and exciter side.
Although based on the PT inspection result is not found of possibility of defect or crack on the retaining ring, however the crack depth testing is performed at several locations that suspected of having crack. In this study, the analog crack depth meter is used to evaluate the depth of crack on the retaining ring. Fig. 8 shows the display of analog crack depth meter. Based on this inspection, there is no find any crack on both sides of the retaining ring.

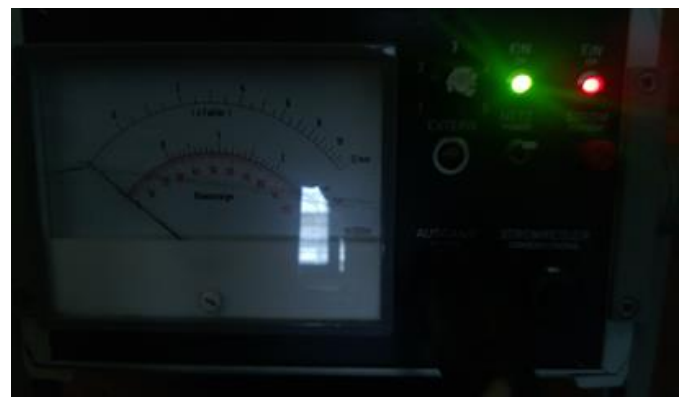

Fig. 8. The display of analog crack depth meter.

The possibility of defect at the shrink fit area and the inner surface of the retaining ring is detected using Ultrasonic Testing (UT). In this study, it uses Krautkramer ultrasonic flaw detector type Epoch 600 with the probe straight beam $0^{\circ}$ and the couplant type grease. Fig. 9(a) shows the UT inspection on the retaining ring. The inspection was applied on the entire of retaining ring in circumferential direction up to 360 degrees with numbering identity on both side i.e. exciter side and turbine side, see Fig. 9(b). Based on the inspection using UT, there is no detected anomaly the shrink fit area and the inner surface of the retaining ring.

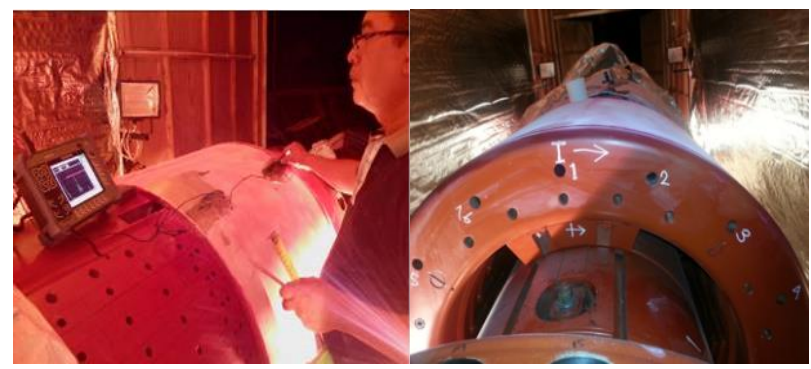

(a)

(b)

Fig. 9. (a) The UT inspection and (b) numbering identity at circumferential direction.

\section{CONCLUSION}

It has been done the in-situ inspection of the retaining ring without removing the retaining ring from the generator rotor, The results of the inspection of 4 [four] of the NDT method was not detected the presence of defects on both sides of the retaining ring generator (turbine and exciter side), so it can't be done further study the remaining life of the retaining ring as a result of the growth defect / crack. Therefore, it can be concluded that the retaining ring is not degraded and the service life of components is still appropriate with the specifications of the manufacture. Although based on the results of the assessment and inspections on the retaining ring, it did not find any defects, but the inspection and checking the possibility of defects due to the influence of the next operation must be done periodically based on the recommendations of the manufacturer. 


\section{ACKNOWLEDGMENT}

The authors would like to thank to PT. Geo Dipa Energy and all the team members of energy research, Indonesian Institute of Sciences for any assistance that has been given.

\section{REFERENCES}

[1] Working Group A1.16, Conseil Internationale Des Grands Reseaux Electriques, Generator End-Winding Retaining Rings-a Literature Survey and Care Guideline, CIGRE Publication, 2010.

[2] S. H. Hwang and J. S. Choi, "Analysis of Shrink-Fitted Retaining Ring on Rotor Body in Generator," in Proc. ABAQUS Users' Conference, 2004, pp. 359-372.

[3] R. Gutkin and B. Alfredsson, "Growth of fretting fatigue cracks in a shrink-fitted joint subjected to rotating bending," Engineering Failure Analysis, vol. 15, pp. 582-596, 2008.

[4] N. Antoni, "Contact separation and failure analysis of a rotating thermo-elastoplastic shrink-fit assembly," Applied Mathematical Modelling, vol. 37, pp. 2352-2363, 2013.

[5] J. Juoksukangas, A. Lehtovaara, and A. Mäntylä, "The effect of contact edge geometry on fretting fatigue behavior in complete contacts," Wear, vol. 308, pp. 206-212, 2013.

[6] T. Juuma, "Torsional fretting fatigue strength of a shrink-fitted shaft with a grooved hub," Tribology International, vol. 33, pp. 537-543, 2000.

[7] B. Alfredsson, "Fretting fatigue of a shrink-fit pin subjected to rotating bending: Experiments and simulations," International Journal of Fatigue, vol. 31, pp. 1559-1570, 2009.

[8] D. Hao and D. Wang, "Finite-element modeling of the failure of interference-fit planet carrier and shaft assembly," Engineering Failure Analysis, vol. 33, pp. 184-196, 2013.

[9] F. Zeng, Z. B. Cai, Z. Q. Feng, and Z. R. Zhou, "In situ observation and analysis of a shrink-fitted PMMA shaft subjected to rotating bending," Tribology International, vol. 48, pp. 149-154, 2012.

[10] F. Lanoue, A. Vadean, and B. Sanschagrin, "Fretting fatigue strength reduction factor for interference fits," Simulation Modelling Practice and Theory, vol. 19, pp. 1811-1823, 2011.

[11] J. Stein, "Retaining ring cracking at wisconsin electric power company's port Washington unit 1 root cause analysis," Electric Power Research Institute, 2002.

[12] J. Stein, "Retaining ring failure at Comanche unit 2: root cause of analysis," Electric Power Research Institute, 1996.

[13] Testing instrument manufacturer. (2014). crack depth gauge. [Online]. Available: http://www.tester-supplier.com/11-2-crack-depth-gauge.ht $\mathrm{ml}$

[14] National instruments. (2014). Fundamentals of Ultrasonic Imaging and Flaw Detection. [Online]. Available: http://www.ni.com/white-paper/3368/en/

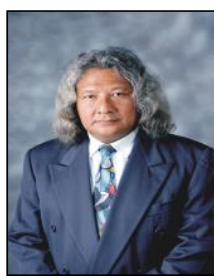

Imam Djunaedi received his B.S. degree in mechanical engineering from Institut Teknologi Bandung, Indonesia in 1984. He had been research as a Ph.D. research student at School of Computer \& Electrical Engineering Curtin University of Technology, Australia from 1993 to 1996 . He is working as a researcher at Indonesian Institute of Sciences (LIPI) from 1986 until now. His research areas are renewable energy, optimization, mechatronics, thermal engineering and power plant.

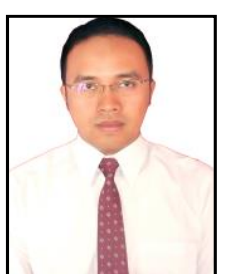

fluid dynamics.
Hilman Syaeful Alam received his B.S. degree in mechanical engineering from Jenderal Achmad Yani University, Indonesia in 2003 and M.S. degree in mechanical engineering from Institut Teknologi Bandung, Indonesia in 2010. He is working as a researcher at Indonesian Institute of Sciences (LIPI) from 2006 until now. His research areas are renewable energy, engineering design, solid mechanics, instrumentation, finite element and computational 

Clean Coal Technology 
